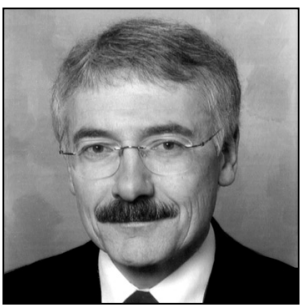

Friedrich Schoch

Prof. Dr. jur.

Albert-Ludwigs-Universität Freiburg

\title{
Zur Europäisierung des Verwaltungsrechts
}

\section{Einführung in die Thematik}

Die „Determinanten der modernen Staatsverwaltung” - so die Thematik des V. Abschnitts unseres Tagungsthemas „Anfänge der modernen Staatsverwaltung” - müssen die europäische Perspektive einbeziehen, wollen sie nicht defizitär sein. Aus juristischer Sicht zwingt dies zu einer Analyse der Europäisierung der innerstaatlichen Rechtsordnung. Treffend ist dieser (noch lange nicht abgeschlossene) Prozess ${ }^{* 1}$ als ein „Fundamentalvorgang” charakterisiert worden: die Verwandlung einer autonomen Rechtsordnung in die Rechtsordnung eines Mitgliedstaates. ${ }^{*}$

\section{Omnipräsenz des Unionsrechts}

Dieser Vorgang ist umfassend angelegt. Er umgreift nicht nur einzelne Rechtsgebiete (z.B. Wettbewerbsrecht, Verbraucherschutzrecht, Umweltrecht), sondern die gesamte Rechtsordnung (Zivilrecht, Staats- und Verwaltungsrecht, Strafrecht). ${ }^{*}$ Die Europäisierung der innerstaatlichen Rechtsordnung erfasst zudem alle Dimensionen des Rechts: Rechtsetzung und Rechtsanwendung sind angesprochen, Gesetzgebung sowie Verwaltung und Rechtsprechung sind betroffen, Inhalte des Rechts und Methoden des Rechts werden herausgefordert, das Recht als Instrument und das Recht in seinem Selbststand (gegenüber der Politik) werden aufgerufen, unterschiedliche Rationalitäten im Rechtsverständnis prallen aufeinander. ${ }^{*}$

In der Sache konzentrieren sich die nachfolgenden Überlegungen auf das Allgemeine Verwaltungsrecht sowie auf das Verwaltungsverfahrensrecht und das Verwaltungsprozessrecht. Auf diesen Rechtsgebieten lassen sich signifikante Europäisierungsmuster nachweisen und bemerkenswerte Eigenrationalitäten des EURechts aufzeigen. Der Zugriff auf die erwähnten Materien dient zugleich der Begrenzung des Rechtsstoffes und erleichtert die Verständigung, da Spezifika des Besonderen Verwaltungsrechts ${ }^{*} 5$ ausgeblendet bleiben.

Eine Chronologie der Europäisierung des innerstaatlichen Rechts bietet A. K. Mangold. Gemeinschaftsrecht und deutsches Recht. Tübingen: Mohr Siebeck 2011, S. $187 f f$.

2 R. Wahl. Herausforderungen und Antworten: Das Öffentliche Recht der letzten fünf Jahrzehnte. Berlin: de Gruyter 2006, S. $94 f f$.

3 Überblick dazu bei F. Schoch. Impulse des Europäischen Gemeinschaftsrechts für die Fortentwicklung der innerstaatlichen Rechtsordnung. - Verwaltungsblätter für Baden-Württemberg (VBlBW) 2003, S. $297 \mathrm{ff}$.

4 Näher dazu U. Mager. Entwicklungslinien des Europäischen Verwaltungsrechts. - P. Axer u.a. (Hrsg.). Das Europäische Verwaltungsrecht in der Konsolidierungsphase. - Die Verwaltung Beiheft 2010/10, S. 11 ff.; W. Kahl. Die Europäisierung des Verwaltungsrechts als Herausforderung an Systembildung und Kodifikationsidee. - Ibid., S. 39ff;; O. Lepsius. Hat die Europäisierung des Verwaltungsrechts Methode? Oder: Die zwei Phasen der Europäisierung des Verwaltungsrechts. - Ibid., S. 179ff.

5 Umfassende Darstellung einzelner Gebiete des Besonderen Verwaltungsrechts in den Einzelbeiträgen der $\S \S 16 f f$. in R. Schulze, M. Zuleeg, S. Kadelbach (Hrsg.). Europarecht. 2. Aufl. Baden-Baden: Nomos 2010 und in P. Terhechte (Hrsg.). Verwaltungsrecht der Europäischen Union. Baden-Baden: Nomos 2011. 


\section{Begriff und Funktion der „Europäisierung”}

Der Begriff „Europäisierung” des Rechts wird hier in einem engen Sinne ${ }^{* 6}$ verstanden und meint den Prozess fortschreitender Beeinflussung, Wandlung und Überformung eines Rechtsgebietes durch die Rechtsmassen des EU-Rechts und durch das in ihnen wirksame Rechtsdenken. ${ }^{*} 7$ Unberücksichtigt bleibt das Recht der EMRK. Keine Beachtung finden auch die ebenenübergreifenden Verwaltungsstrukturen zwischen europäischen und mitgliedstaatlichen Verwaltungsbehörden, wie sie etwa in der gegenseitigen Anerkennung von Verwaltungsentscheidungen und dem Handeln der Europäischen Agenturen zum Ausdruck kommen.. ${ }^{*}$

Die Konzentration auf die vertikale Perspektive zwischen EU-Recht und mitgliedstaatlichem Recht ist verschiedentlich als zu eng kritisiert worden. Der Begriff „Europäisierung” müsse auch auf die Rechtsbildungsprozesse im Unionsrecht erstreckt werden, damit die Einflüsse aus dem mitgliedstaatlichen Recht in das werdende EU-Recht nicht länger ausgeblendet bleiben. ${ }^{*}{ }^{*}$ In der Sache muss diesem erweiterten Begriffsverständnis nicht widersprochen werden. Der juristische Wert der auf die Überformung des nationalen Verwaltungsrechts durch das Unionsrecht gerichteten Betrachtungsweise wird, wie zu zeigen sein wird, durch jenes extensive Begriffsverständnis in keiner Weise geschmälert.

Der hier gewählte Problemzugang findet eine sichere Verankerung im positiven Recht. Der Grundsatz des indirekten Vollzugs von EU-Recht ist mit dem Vertrag von Lissabon im primären Unionsrecht festgeschrieben worden ${ }^{* 10}$ und besagt, dass mitgliedstaatliche Behörden das Unionsrecht nach Maßgabe des innerstaatlichen Verwaltungs(verfahrens)rechts zur Anwendung bringen. ${ }^{*}{ }^{* 11}$ Im Rechtsschutzverfahren obliegt die Verwaltungskontrolle dem nationalen Richter, der als „Unionsrichter” fungiert. ${ }^{*}{ }^{12}$

Grundlage dieses Konzepts ist die Verfahrensautonomie der EU-Mitgliedstaaten. Die Europäische Union hat, dem Prinzip der begrenzten Einzelermächtigung verpflichtet (Art. 5 Abs. 1 S. 1 und Abs. 2 EUV), für das Allgemeine Verwaltungsrecht, das Verwaltungsverfahrensrecht und das Verwaltungsprozessrecht keine generelle Regelungskompetenz. Die Vollzugs- und Kontrollinstrumente in Bezug auf das EU-Recht sind dem innerstaatlichen Recht zu entnehmen. Gesprochen wird seit jeher von der institutionellen und verfahrensmäßigen Autonomie der Mitgliedstaaten. ${ }^{*}{ }^{13}$

Bei einem solchen Modell sind die Risiken für die einheitliche reale Geltung des Unionsrechts geradezu systemimmanent. Der der Wahrung des Rechts (Art. 19 Abs. 1 S. 2 EUV) verpflichtete Europäische Gerichtshof (EuGH) hat indessen nie einen Zweifel daran gelassen, dass die einheitliche Anwendung des Unionsrechts ein Grundprinzip der Europäischen Union darstellt. ${ }^{*} 4$ Hierin findet die „Europäisierung” der mitgliedstaatlichen Rechtsordnungen gleichsam ihre Legitimität.

6 Vgl. zu weiteren Europäisierungsbegriffen T. Siegel. Europäisierung des Öffentlichen Rechts. Tübingen: Mohr Siebeck 2012, Rdn. $71 f f$.

7 Im Anschluss an E. Schmidt-Aßmann. Zur Europäisierung des allgemeinen Verwaltungsrechts. - P. Badura (Hrsg.). Wege und Verfahren des Verfassungslebens: Festschrift für Peter Lerche zum 65. Geburtstag. München: Beck 1993, S. 513.

8 Dazu E. Schmidt-Aßmann. Perspektiven der Europäisierung des Verwaltungsrechts. - P. Axer u.a. (Hrsg.) (Fn. 4), S. 268ff.

$9 \quad$ R. Wahl. Europäisierung: Die miteinander verbundenen Entwicklungen von Rechtsordnungen als ganzen. - H.-H. Trute u.a. (Hrsg.). Allgemeines Verwaltungsrecht - zur Tragfähigkeit eines Konzepts. Tübingen: Mohr Siebeck 2008, S. 869ff.; R. Wahl. Die Rechtsbildung in Europa als Entwicklungslabor. - Juristenzeitung (JZ) 2012, S. 861 ff. - DOI: http://dx.doi. org/10.1628/002268812802866678.

10 Vertrag über die Arbeitsweise der Europäischen Union (AEUV) (konsolidierte Fassung). - Amtsblatt der Europäischen Union (ABl. EU), 9.5.2008, C 115. Art. 291 Abs. 1: Die Mitgliedstaaten ergreifen alle zur Durchführung der verbindlichen Rechtsakte der Union erforderlichen Maßnahmen nach innerstaatlichem Recht.

11 C. Haselmann. Delegation und Durchführung gemäß Art. 290 und 291 AEUV. Berlin: Duncker \& Humblot 2012, S. 171 ff.

12 Dazu E. Schmidt-Aßmann, W. Schenk. - F. Schoch, J.-P. Schneider, W. Bier (Hrsg.). Verwaltungsgerichtsordnung. München: Beck (Losebl.-Ausg.), Einleitung (3.2014) Rdn. 109.

13 G. C. Rodríguez Iglesias. Zu den Grenzen der verfahrensrechtlichen Autonomie der Mitgliedstaaten bei der Anwendung des Gemeinschaftsrechts. - Europäische Grundrechte-Zeitschrift (EuGRZ) 1997, S. 289; S. Kadelbach. Allgemeines Verwaltungsrecht unter europäischem Einfluss. Tübingen: Mohr Siebeck 1999, S. 110 ff.; aktuell zu der Thematik C. Krönke. Die Verfahrensautonomie der Mitgliedstaaten der Europäischen Union. Tübingen: Mohr Siebeck 2013, S. $37 f f$.

14 Vgl. etwa EuGH, Urteil v. 14.2.2012, C-204/o9, Flachglas Torgau vs. Bundesrepublik Deutschland. - Neue Zeitschrift für Verwaltungsrecht (NVwZ) 2012, 491 Rdn. 37; T. v. Danwitz. Europäisches Verwaltungsrecht. Berlin/Heidelberg: Springer 2008, S. 155ff:: Einheitlichkeit des Gemeinschaftsrechts als „Konstitutionsprinzip” der Rechtsordnung. 


\section{Mechanismen und Eigenrationalitäten des EU-Rechts}

Bevor den Rechtswirkungen der Europäisierung in der innerstaatlichen Rechtsordnung nachgegangen wird, lohnt ein Blick auf allgemeine Mechanismen und Eigenrationalitäten des EU-Rechts, die den Prozess der „Europäisierung” entfachen und beschleunigen. Grundlage der Analyse ist die Anerkennung der supranationalen Qualität des Unionsrechts, ${ }^{*} 5$ das sich insoweit kategorial (und nicht nur graduell) vom überkommenen Völkerrecht unterscheidet.

Vor diesem Hintergrund haben sich die folgenden - nicht abschließend zu verstehenden - fünf Dogmen als besonders wirkungsvoll erwiesen:

- Der (grundsätzliche) Vorrang des EU-Rechts gegenüber dem Recht der Mitgliedstaaten ${ }^{* 16}$ wirkt als Anwendungsvorrang ${ }^{* 17}$ (nicht als Geltungsvorrang). Im Konfliktfall darf kollidierendes innerstaatliches Recht nicht angewendet werden. ${ }^{*} 18$

- Die unionsrechtskonforme Auslegung des innerstaatlichen Rechts ist eine längst anerkannte Interpretationsmethode, die den schonenden Ausgleich zwischen der supranationalen und der innerstaatlichen Rechtsordnung herstellt. ${ }^{*} 9$ Am geläufigsten ist der Unterfall der richtlinienkonformen Auslegung des nationalen Rechts. ${ }^{{ }_{20}}$

- Die unmittelbare Wirkung (unmittelbare Anwendung) von Normen des Unionsrechts im innerstaatlichen Rechtskreis gehört auch jenseits der Grundfreiheiten mittlerweile zu den juristischen Selbstverständlichkeiten. Dazu zählt insbesondere die vom Gerichtshof gegen das positive Recht (Art. 288 Abs. 3 AEUV, zuvor Art. 249 Abs. 3 EGV) durchgesetzte unmittelbare Wirkung von (an die Mitgliedstaaten adressierten) Richtlinien. ${ }^{* 21}$

- Der Äquivalenzgrundsatz und der Effektivitätsgrundsatz setzen der Verfahrensautonomie der Mitgliedstaaten in den von der Europäischen Union mangels Rechtsetzungskompetenz nicht geregelten Bereichen Grenzen. ${ }^{* 2}$ Ein geradezu unerschöpflicher „Speicher” für unendlich viele Deduktionen ist der Effektivitätsgrundsatz, wonach mitgliedstaatliche Verfahren die Ausübung der durch die Unionsrechtsordnung verliehenen Rechte nicht praktisch unmöglich machen oder übermäßig erschweren dürfen. ${ }^{{ }^{2} 3}$

- Die vom Gerichtshof seit einiger Zeit postulierte Kohärenz im europäischen Rechts(schutz)system $^{{ }^{*} 24}$ setzt dem vertragsrechtlichen Gebot der begrenzten Einzelermächtigung (Art. 5 Abs. 1 S. 1 ,

15 Bundesverfassungsgericht (BVerfG), Urteil v. 30.6.2009, 2 BvE 2/o8. - Entscheidungen des Bundesverfassungsgerichtes (BVerfGE) 123, 267 (348f., 356 f., 364, 379, 381).

16 Näher dazu D. Ehlers. Der Vorrang des Europäischen Unionsrechts. - Juristische Ausbildung (Jura) 2011, S. 187ff.; M. Polzin. Das Rangverhältnis von Verfassungs- und Unionsrecht nach der neuesten Rechtsprechung des BVerfG. - Juristische Schulung (JuS) 2012, S. 1 ff. Zu möglichen Ausnahmen D. Ehlers, A. Eggert. Zur Zulässigkeit einer zeitlich begrenzten weiteren Anwendung gemeinschaftsrechtswidrigen nationalen Rechts. - JZ 2008, S. $585 \mathrm{ff}$.

17 BVerfG, Urteil v. 7.9.2011, 2 BvR 987/10 u.a. - BVerfGE 123, 267 (400) anerkennt „das Institut des Anwendungsvorrangs” als Produkt der Rechtsprechung des EuGH.

18 Aus der Praxis z.B. Bundesverwaltungsgericht (BVerwG), Urteil v. 16.5.2013, 5 C 22.12. - Neue Juristische Wochenschrift (NJW) 2013, 2919 (2921): Unvereinbarkeit einer gesetzlichen Regelung zur Ausbildungsförderung bei einem Auslandspraktikum mit dem unionsrechtlichen Freizügigkeitsrecht (Art. 20 Abs. 2 lit. a, 21 Abs. 1 AEUV) führt mangels einer möglichen unionsrechtskonformen Auslegung zu einem Anwendungsverbot des $\S 5$ Abs. 5 S. 1 Hs. 2 Bundesausbildungsförderungsgesetz.

19 M. Brenner. Der Gestaltungsauftrag der Verwaltung in der Europäischen Union. Tübingen: Mohr Siebeck 1996, S. $92 f$.; vgl. ferner J. Antweiler. Die Auslegungsmethoden des Gerichtshofs der Europäischen Gemeinschaften. Frankfurt a. Main: Lang 1997, S. 74ff.

20 Nach BVerfG, Beschluss v. 26.9.2011, 2 BvR 2216/o6 u.a. - NJW 2012, 669, verpflichtet der aus Art. 4 Abs. 3 EUV folgende Grundsatz der Unionstreue alle mitgliedstaatlichen Stellen zur richtlinienkonformen Auslegung des nationalen Rechts (Rdn 46); Grenzen ergäben sich aus „dem nach innerstaatlicher Rechtstradition methodisch Erlaubten”, so dass „eine Auslegung des nationalen Rechts contra legem" ausscheide (Rdn. 47).

21 Vgl. etwa (mit weiteren Nachweisen (m.w.N.) zu seiner Rechtsprechung) EuGH, Urteil v. 12.7.2012, C-55/11, C-57/11, C-58/11, Vodafone España und France Telecom España. - NVwZ 2012, 1303 Rdn. 37: Der Einzelne kann sich „in all den Fällen, in denen die Bestimmungen einer Richtlinie inhaltlich unbedingt und hinreichend genau sind, vor den nationalen Gerichten gegenüber dem Staat auf diese Bestimmungen berufen, wenn der Staat die Richtlinie nicht fristgemäß oder unzulänglich in nationales Recht umgesetzt hat".

22 Ausführlich zu der Thematik J. König. Der Äquivalenz- und Effektivitätsgrundsatz in der Rechtsprechung des Europäischen Gerichtshofs. Baden-Baden: Nomos 2011, S. 92 ff. und S. 105ff.

23 Vgl. etwa (m.w.N. zu seiner Rechtsprechung) EuGH, Urteil v. 15.1.2013, C-416/10. - Zeitschrift Natur und Recht (NuR) 2013, 113 Rdn. 85.

24 EuGH, Urteil v. 6.12.2005, C-461/03. - Sammlung der Rechtsprechung (Slg.) 2005, I-10513 Rdn. 22; Darstellung und Kritik der EuGH-Rechtsprechung bei F. Schoch. Gerichtliche Verwaltungskontrollen. - W. Hoffmann-Riem, E. Schmidt-Aßmann, 
Abs. 2 EUV) die richterrechtliche Neigung zur Unitarisierung weiter Teile der innerstaatlichen Rechtsordnung entgegen. ${ }^{* 25}$

Die skizzierten Mechanismen und Eigenrationalitäten des EU-Rechts, die sämtlich im Dienste der „Europäisierung” stehen, müssen als allgemeine, „vor die Klammer gezogene” Struktursicherungen verstanden werden, die auf jedem Rechtsgebiet Anwendung finden können. Die fassbaren Ergebnisse der „Europäisierung" der innerstaatlichen Rechtsordnung entstehen demnach durch eine juristische Interpretationsleistung, die strukturelle Vorgaben des EU-Rechts mit der inhaltlichen Ausrichtung nationaler Rechtsnormen auf das Unionsrecht verknüpft.

\section{Allgemeines Verwaltungsrecht}

Die Funktionslogik des EU-Rechts in bestimmten Anwendungsfeldern kann zunächst im (materiellen) Allgemeinen Verwaltungsrecht aufgezeigt werden. ${ }^{* 26}$ Für dieses Rechtsgebiet (als solches) hat die Europäische Union nach der geltenden Zuständigkeitsordnung keine Regelungskompetenz. Gleichwohl belegen die folgenden drei ausgewählten Beispiele die Eindringtiefe der supranationalen Rechtsordnung in den innerstaatlichen Rechtskreis.

\section{Subjektives öffentliches Recht}

Die Rechtsstellung des Einzelnen im Gemeinwesen wird maßgeblich durch das subjektive öffentliche Recht konstituiert. Herausragend sind insoweit die Grundrechte des nationalen Verfassungsrechts, die Grundfreiheiten des primären EU-Rechts und die Grundrechte der seit Dezember 2009 in Kraft befindlichen Europäischen Grundrechte-Charta. Im Alltag der Verwaltung dominieren indessen die subjektiven öffentlichen Rechte des Gesetzesrechts.

\section{a) Herkömmliches deutsches Konzept}

Gewonnen werden diese Rechte nach überkommener deutscher Rechtsauffassung anhand der Schutznormtheorie. ${ }^{* 27}$ Danach ist zu erforschen, ob eine Rechtsnorm (neben ihrer objektiv-rechtlichen Zwecksetzung) zumindest auch dem Schutz von Individualinteressen zu dienen bestimmt ist und dem Träger des Individualinteresses die Rechtsmacht einräumt, von dem Normverpflichteten die Einhaltung der Rechtsnorm verlangen zu können. ${ }^{* 28}$ In dem Kanon von Methoden und Regeln zur Erschließung des individualschützenden Gehalts einer Norm ${ }^{* 29}$ verfügt der Richter (als Letztinterpret) über nicht unerhebliche Auslegungsspielräume. Das Ergebnis bestimmter Rechtsprechungsentwicklungen in Deutschland ist frappierend: Während z.B. der Nachbarschutz im Baurecht sukzessive ausgebaut worden ist, fristet der Konkurrentenschutz im Wirtschaftsverwaltungsrecht ein eher kümmerliches Dasein. ${ }^{*}{ }^{0}$ In Verbundbegriffen zusammengefasste

A. Voßkuhle (Hrsg.). Grundlagen des Verwaltungsrechts. Bd. III. 2. Aufl. München: Beck 2013, § 50 Rdn. $26 f f$.

25 E. Schmidt-Aßmann. Kohärenz und Konsistenz des verwaltungsgerichtlichen Rechtsschutzes. - Die Verwaltung 2011, S. 105ff.; E. Schmidt-Aßmann. Der Kohärenzgedanke in den EU-Verträgen: Rechtssatz, Programmsatz oder Beschwörungsformel? I. Appel, G. Hermes, C. Schönberger. Festschrift für Rainer Wahl zum 70. Geburtstag. Berlin: Duncker \& Humblot 2011, S. $819 f f$.

26 Frühzeitig dazu E. Schmidt-Aßmann. Deutsches und Europäisches Verwaltungsrecht - Wechselseitige Einwirkungen. Deutsches Verwaltungsblatt (DVBl.) 1993, S. 924ff.; F. Schoch. Die Europäisierung des Allgemeinen Verwaltungsrechts. JZ 1995, S. $109 f f$.

27 H. Bauer. Altes und Neues zur Schutznormtheorie. - Archiv des öffentlichen Rechts (AöR) (113) 1988, S. 582 ff.; H. Bauer. Die Schutznormtheorie im Wandel. - D. Heckmann, K. Meßerschmidt (Hrsg.). Gegenwartsfragen des Öffentlichen Rechts. Berlin: Duncker \& Humblot 1988, S. 113ff.; J. Pietzcker. Die Schutznormlehre. - Festschrift für Josef Isensee. Heidelberg: Müller 2007, S. $577 f f$.

28 BVerwG, Urteil v. 24.9.1998, 4 CN 2.98. - Entscheidungen des Bundesverwaltungsgericht (BVerwGE) 107, 215 (220); 111, 276 (280); Niedersächsisches Oberverwaltungsgericht, Beschluss v. 6.5.2011, 1 ME 14/11. - DVBl. 2011, 1487 (1488); Oberverwaltungsgericht Nordrhein-Westfalen, Beschluss v. 19.3.2009, 13 A 476/o8 u.a. - NVwZ 2009, 1383.

29 E. Schmidt-Aßmann. - T. Maunz, G. Dürig (Begründer). Grundgesetz. München: Beck (Losebl.-Ausg.), Art. 19 Abs. 4 (2003) Rdn. 128.

30 Die jeweilige Rechtsprechungsentwicklung ist nachgezeichnet bei F. Schoch. - W. Hoffmann-Riem, E. Schmidt-Aßmann, A. Voßkuhle (Hrsg.) (Fn. 24), § 50 Rdn. 140 ff. 
aggregierte Individualinteressen (z. B. „Verbraucherschutz”) finden nach überkommener Doktrin keine subjektivrechtliche Anerkennung. ${ }^{*}{ }^{3}$

\section{b) Funktionale Subjektivierung nach Europarecht}

Die Funktionslogik des EU-Rechts folgt im vorliegenden Zusammenhang dem Konzept der funktionalen Subjektivierung; der Einzelne wird in den Dienst einer wirksamen Durchsetzung des Unionsrechts gestellt und damit in die dezentrale Vollzugskontrolle einbezogen. ${ }^{*}{ }^{32}$ Individuelle Rechte werden bereits dann anerkannt, wenn und soweit eine Rechtsnorm auf die Verleihung von Rechten an den Einzelnen zielt. ${ }^{*} 3$ Damit lehnt auch das Europarecht einen allgemeinen Normvollziehungsanspruch ab und rezipiert die Schutznormtheorie als Strukturmodell für die Unterscheidung zwischen individuellen Berechtigungen und Allgemeininteressen. ${ }^{*} 34$ Inhaltlich erfolgt jedoch eine Ausweitung sowohl in Bezug auf den Schutzgegenstand als auch auf den geschützten Personenkreis, indem die normative Bündelung von Einzelinteressen die individuelle Berechtigung nicht entfallen lässt.

So genügt im Umweltrecht der Schutz der „Volksgesundheit” bzw. „öffentlichen Gesundheit” für die Bejahung der individualschützenden Wirkung einer Rechtsnorm, ${ }^{*} 35$ im Verbraucherschutzrecht werden individuelle Rechte bereits anerkannt, wenn der Rechtsakt auf den Schutz der „Verbraucher(schaft)” zielt. ${ }^{*} 6$ Die aktuelle rechtswissenschaftliche Diskussion rankt - bei Anerkennung der funktionalen Subjektivierung - darum, ob der Schutz personaler Rechtsgüter Voraussetzung für die individualschützende Wirkung einer Rechtsnorm ist ${ }^{*} 37$ oder ob eine Erweiterung der Schutznormtheorie angezeigt ist, die auch die Ermächtigung zur individuellen Durchsetzung öffentlicher Interessen umfasst. ${ }^{*}{ }^{38}$ Die praktischen Konsequenzen der Europäisierung des subjektiven öffentlichen Rechts zeigen sich vor allem auf dem Gebiet des Verwaltungsprozessrechts im Rahmen der Klagebefugnis.

\section{Rücknahme von Verwaltungsakten}

Als Handlungsform der Verwaltung kommt dem Verwaltungsakt im Verhältnis des Staates zum Einzelnen eine wichtige stabilisierende Wirkung zu. Im Falle der Bestandskraft ist der Verwaltungsakt die Grundlage für den Vertrauensschutz des Individuums in die Beständigkeit der staatlichen Entscheidung. Beim begünstigenden, aber rechtswidrigen Geldleistungsbescheid kollidieren der Vertrauensschutz des Begünstigten und das Prinzip der Gesetzmäßigkeit der Verwaltung. Nach deutschem Recht ist die zuständige Behörde an der Rücknahme des Verwaltungsakts gehindert, wenn sich der Vertrauensschutz unter bestimmten gesetzlichen Voraussetzungen ( $\$ 8$ Abs. 1 S. 2, Abs. 2, Abs. 4 VwVfG) durchsetzt.

\section{a) Wettbewerbsrecht versus Vertrauensschutz}

Im Falle europarechtswidriger staatlicher Beihilfen (Subventionen), insbesondere wegen Verstoßes gegen Art. 108 Abs. 2 und 3 AEUV (zuvor Art. 88 Abs. 2 und 3 EGV), erfolgen die Rücknahme des Bewilligungsbescheids und die Rückforderung der Geldleistung mangels europarechtlicher Befugnisnormen nach nationalem Recht. Zu beachten ist indes das europarechtliche Effektivitätsgebot. Auf dieser Grundlage hat das

${ }^{31} \quad$ R. Wahl. - F. Schoch, J.-P. Schneider, W. Bier (Hrsg.) (Fn. 12), Vorb. § 42 Abs. 2 Rdn. $55 f f$.

32 J. Masing. Der Rechtsstatus des Einzelnen im Verwaltungsrecht. - W. Hoffmann-Riem, E. Schmidt-Aßmann, A. Voßkuhle (Hrsg.). Grundlagen des Verwaltungsrechts. Bd. I. 2. Aufl. München: Beck 2012, § 7 Rdn. $91 f f$.

33 EuGH, Urteil v. 17.10.1991, C-58/89. - Slg. 1991, I-4983 Rdn. 14 (auch in: NVwZ 1992, 459 Rdn. 14); EuGH, Urteil v. 8.10.1996, C-178/94 u.a.. - Slg. 1996, I-4845 (auch in: NJW 1996, 3141 Rdn. 37ff.); EuGH, Urteil v. 17.4.2007, C-470/o3. - Slg. 2007, I-2749 Rdn. 78 und 79 (auch in: EuZW 2007, 480 Rdn. 78 und 79; NVwZ 2007, 1282 Rdn. 78 und 79).

34 G. C. Rodríguez Iglesias. Gedanken zum Entstehen einer Europäischen Rechtsordnung. - NJW 1999, S. 7 bemerkt, der EuGH habe die Schutznormtheorie übernommen.

35 EuGH, Urteil v. 25.7.2008, C-237/07, Janecek. - Slg. 2008, I-6221 Rdn. 37 und 38 (auch in: EuZW 2008, 573 Rdn. 37 und 38; NVwZ 2008, 948 Rdn. 37 und 38 mit Zusammenfassung der bisherigen Judikatur).

36 EuGH, C-178/94 u.a. (Fn. 33), Rdn. 36, 41. Gegenbeispiel: EuGH, Urteil v. 12.10.2004, C-222/o2. - Slg. 2004, I-9425 (auch in: EuZW 2004, 689 (m. Bespr. Häde EuZW 2005, 39); NJW 2004, 3479): kein Schutz der Einleger im Bankenaufsichtsrecht.

37 F. Schoch. - W. Hoffmann-Riem, E. Schmidt-Aßmann, A. Voßkuhle (Hrsg.) (Fn. 24), § 50 Rdn. $159 f$.

38 M. Hong. Subjektive Rechte und Schutznormtheorie im europäischen Verwaltungsrechtsraum. - JZ 2012, S. 384ff. 
nationale Recht ( $\S$ 48, 49a VwVfG) durch die Rechtsprechung des EuGH eine komplette supranationale Durchdringung erfahren: ${ }^{*} 39$

- Die nach deutschem Recht bestehende gesetzliche Vermutung für den Vertrauensschutz nach dem Verbrauch der gewährten Leistung durch den Begünstigten (§ 48 Abs. 2 S. 2 VwVfG) wird durch das Europarecht widerlegt.

- Die Jahresfrist für die Rücknahme des begünstigenden Verwaltungsakts (§ 48 Abs. 4 S. 1 VwVfG) wird durch das supranationale Recht derogiert, weil andernfalls die Rückforderung der Beihilfe (Subvention) praktisch unmöglich werden könnte.

- Das Rücknahmeermessen der Behörde (§ 48 Abs. 1 S. 1 VwVfG) wird europarechtlich nicht anerkannt, weil bei einer Ermessensbetätigung zu Gunsten des Begünstigten der Rechtsgrund für die erlangte Geldleistung bestehen bliebe und ein staatlicher Rückforderungsanspruch daher nicht durchsetzbar wäre.

- Nach erfolgter Rücknahme des Bewilligungsbescheids ist gegenüber dem Rückforderungsanspruch des Staates der Entreicherungseinwand des Begünstigten (§ 49a Abs. 2 VwVfG) europarechtlich abgeschnitten.

\section{b) Instrumentelle Funktion des nationalen Verwaltungsrechts}

Im Ergebnis führen die Einwirkungen des Unionsrechts auf das nationale Recht zu einer Denaturierung der $\S \S 48,49 \mathrm{a} \mathrm{VwVfG} .{ }^{*}{ }^{40}$ Das innerstaatliche (deutsche) Recht hat seinen Eigenwert verloren und steht nur noch in der Pflicht, die unionsrechtlichen Vorgaben zu vollziehen. ${ }^{* 1}$ An diesem Effekt der „Europäisierung” hat sich auch nach Erlass der europäischen Beihilfeverordnung ${ }^{*} 42$ nichts geändert. ${ }^{*} 43$ Schon zur Vermeidung von Wettbewerbsverzerrungen im Binnenmarkt muss sich das Unionsrecht mit dem Ziel einer Rückforderung rechtswidriger staatlicher Beihilfen durchsetzen. ${ }^{*} 4$ Das BVerwG hat die Judikatur des EuGH rezipiert. ${ }^{* 45}$ Das BVerfG hat festgestellt, die Vorgaben des Europarechts verstießen nicht gegen innerstaatliche Grundsätze des Vertrauensschutzes. ${ }^{*} 6$ Vertrauensschutz gegenüber der Rücknahme begünstigender Verwaltungsakte und der Rückforderung von zu Unrecht gewährten öffentlichen Geldleistungen findet nurmehr nach Maßgabe europarechtlicher Standards („Gutgläubigkeit” des Begünstigten) statt. ${ }^{*} 4$

39 EuGH, Urteil v. 20.3.1997, C-24/95, Alcan II. - Slg. 1997, I-1591 (auch in: EuZW 1997, 276 (m. Anm. Hoenike); EuR 1997, 148 (m. Anm. Berrisch); NVwZ 1998, 45 (m. Bespr. Happe, NVwZ 1998, 26); JZ 1997, 722 (m. Anm. Classen)); bestätigend EuGH, Urteil v. 16.7.1998, C-298/96, Oelmühle Hamburg. - Slg. 1998, I-4767 (auch in: EuZW 1998, 603); EuGH, Urteil v. 3.7.2001, C-378/98. - Slg. 2001, I-5107 (auch in: EuZW 2001, 565; NVwZ 2002, 195).

40 So treffend D. Ehlers. Europäisches Recht und Verwaltungsrecht. - H.-U. Erichsen, D. Ehlers (Hrsg.). Allgemeines Verwaltungsrecht. 14. Aufl. Berlin: de Gruyter 2010, § 5 Rdn. 46.

41 Strukturell findet eine Durchbrechung der Verfahrensautonomie der Mitgliedstaaten auf Grund des Effektivitätsgrundsatzes statt; ausführliche Analyse dazu bei C. Krönke (Fn. 13), S. 246ff., $267 f f$.

42 Verordnung (EG) Nr. 659/1999 des Rates über besondere Vorschriften für die Anwendung von Artikel 108 des Vertrages über die Arbeitsweise der Europäischen Union vom 22. März 1999. - ABl. Nr. L 83/1; zuletzt geändert durch Verordnung (EU) 517/2013. - ABl. Nr. L 158/1; die Rückforderung von Beihilfen ist in Art. 14 geregelt, der allerdings nur das Verhältnis zwischen der Europäischen Union und den Mitgliedstaaten betrifft, so dass sich die Rückforderung von Beihilfen gegenüber dem Begünstigten nach wie vor nach innerstaatlichem Recht richtet.

43 Vgl. nur z.B. EuGH, Urteil v. 12.12.2002, C-209/oo. - Slg. 2002, I-11695 (auch in: EuZW 2003, 110; NVwZ 2003, 338); EuGH, Urteil v. 5.10.2006, C-232/05. - Slg. 2006, I-10097 (auch in: EuZW 2007, 56 (m. Anm. Rosenfeld)); EuG, Urteil v. 12.9.2007, T-196/02, MTU Friedrichshafen. - Slg. 2007, II-505 (auch in: EuZW 2007, 613); EuGH, Urteil v. 20.5.2010, C-210/o9, Scott SA. - Slg. 2010, I-4615 (auch in: EuZW 2010, 585).

44 Das gilt auch, wenn die unionsrechtswidrige Beihilfe durch einen privatrechtlichen Vertrag gewährt worden ist; Bundesgerichtshof (BGH), Beschluss v. 13.9.2012, III ZB 3/12. - NVwZ-Rechtsprechungsreport 2012, 960 (963).

45 BVerwG, Urteil v. 23.4.1998, 3 C 15-97. - BVerwGE 106, 328 (auch in: NJW 1998, 3728).

46 BVerfG, Beschluss v. 17.2.2000, 2 BvR 1210/98. - EuZW 2000, 445 (auch in: NJW 2000, 2015 (m. Bespr. Gündisch, NVwZ 2000, 1125); Bayerische Verwaltungsblätter (BayVBl.) 2000, 655 (m. Anm. Lindner und Bausback)).

47 EuGH, Urteil v. 21.7.2007, C-158/o6, Stichting RoM-projecten. - Slg. 2007, I-5103 Rdn. 24ff. (auch in: EuZW 2007, 438 Rdn. 24ff.); kritisch V. Götz. Der knapp bemessene Vertrauensschutz bei der Rückforderung gemeinschaftsrechtswidriger staatlicher Beihilfen. - Die Ordnung der Freiheit. Festschrift für Christian Starck zum siebzigsten Geburtstag. Tübingen: Mohr Siebeck 2007, S. 555ff. 


\section{Staatshaftung}

\section{a) Haftungsgrundlagen im deutschen Recht}

Nach deutschem Rechtsverständnis ist die Staatshaftung dem Allgemeinen Verwaltungsrecht zuzuordnen. ${ }^{* 48}$ Den Kern des Staatshaftungsrechts bildet seit über 100 Jahren die Amtshaftung; ${ }^{*} 49$ danach hat der Staat (bzw. eine seiner Untergliederungen) den einem Dritten entstandenen Schaden zu ersetzen, ${ }^{*} 50$ wenn dieser Schaden auf einer schuldhaften Verletzung einer dem Dritten gegenüber bestehenden Amtspflicht durch einen Beamten (bzw. sonstigen öffentlichen Bediensteten) beruht. ${ }^{*}{ }^{1}$

Ergänzt wird die Amtshaftung insbesondere durch die Haftungsinstitute des enteignungsgleichen Eingriffs und des enteignenden Eingriffs. ${ }^{* 2}$ Gehaftet wird danach für den rechtswidrigen bzw. rechtmäßigen hoheitlichen Eingriff in eine eigentumsrechtlich geschützte (Vermögens-)Position des Geschädigten (mit der Folge eines „Sonderopfers” für die Allgemeinheit); Verschulden ist nicht erforderlich, zu leisten ist allerdings nur eine Entschädigung (und nicht der volle Schadenersatz). ${ }^{*} 53$

\section{b) Indienststellung der Staatshaftung für die Durchsetzung des Unionsrechts}

Dieser „Sekundärrechtsschutz” hat bei Verstößen eines EU-Mitgliedstaates gegen das Unionsrecht eine vollständige europarechtliche Überformung erfahren. In ständiger Rechtsprechung hebt der Gerichtshof hervor, dass ein europarechtlicher Grundsatz der Haftung des Staates für Schäden, die dem Einzelnen durch dem Staat zuzurechnende Verstöße gegen das Unionsrecht entstehen, dem System der Unionsverträge innewohne und die Staatshaftung unter folgenden Voraussetzungen auslöse: ${ }^{*} 54$

(1) Die unionsrechtliche Norm, gegen die verstoßen worden ist, bezweckt die Verleihung von Rechten an den Geschädigten.

(2) Der Verstoß gegen diese Norm ist hinreichend qualifiziert. ${ }^{*} 5$

(3) Zwischen diesem Rechtsverstoß und dem dem Geschädigten entstandenen Schaden besteht ein unmittelbarer Kausalzusammenhang.

Die Anwendung dieser Voraussetzungen für die Staatshaftung obliegt den nationalen Gerichten. ${ }^{*} 6$

48 Vgl. B. Grzeszick. Staatshaftungsrecht. - H.-U. Erichsen, D. Ehlers (Hrsg.) (Fn. 40), § 43.

$49 \S 839$ Abs. 1 S. 1 BGB: „Verletzt ein Beamter vorsätzlich oder fahrlässig die ihm einem Dritten gegenüber obliegende Amtspflicht, so hat er dem Dritten den daraus entstehenden Schaden zu ersetzen.” Art. 34 S. 1 GG: „Verletzt jemand in Ausübung eines ihm anvertrauten öffentlichen Amtes die ihm einem Dritten gegenüber obliegende Amtspflicht, so trifft die Verantwortlichkeit grundsätzlich den Staat oder die Körperschaft, in deren Dienst er steht.”

50 Die Haftung erfolgt durch Geldersatz (nicht: Naturalrestitution); BGH, Beschluss v. 19.12.1960, GSZ 1/60. - Entscheidungen des Bundesgerichtshofs in Zivilsachen (BGHZ) 34, 99 (104); BGH, Urteil v. 9.10.1997, IIIZR4/97. - BGHZ 137, 11 (26).

51 Ausführlich dazu F. Ossenbühl, M. Cornils. Staatshaftungsrecht. 6. Aufl. München: Beck 2013, S. 11 ff.

52 Grundlegend zur Haftung aus enteignungsgleichem Eingriff BGH, Urteil v. 26.1.1984, III ZR 216/82. - BGHZ 90, 17 und BGH, Urteil v. 28.6.1984, III ZR 35/83. - BGHZ 92, 34; ferner z.B. BGH, Urteil v. 9.12.2004, III ZR 263/04. - BGHZ 161, 305. Zur Haftung aus enteignendem Eingriff BGH, Urteil v. 29.03.1984, III ZR 11/83. - BGHZ 91, 20 und BGH, Urteil v. 23.05.1985, III ZR 39/84. - BGHZ 94, 373; ferner z.B. BGH, Urteil v. 19.1.2006, III ZR 121/05. - BGHZ 166, 37 (auch in: NVwZ 2006, 1086).

53 Einzelheiten dazu bei F. Ossenbühl, M. Cornils (Fn. 51), S. 259ff. (enteignungsgleicher Eingriff) und S. 325ff. (enteignender Eingriff).

54 Grundlegend EuGH, Urteil v. 19.11.1991, C-6/90 und 9/90, Francovich. - Slg. 1991, I-5403 Rdn. 40 (auch in: EuZW 1991, 758 Rdn. 40; NJW 1992, 165 Rdn. 40); ferner EuGH, Urteil v. 5.3.1996, C-46/93 und 48/93, Brasserie du Pêcheur. - Slg. 1996, I-1029 Rdn. 51 (auch in: EuZW 1996, 205 Rdn. 51; NJW 1996, 1267 Rdn. 51); aus jüngerer Zeit z.B. EuGH, Urteil v. 24.3.2009, C-445/o6, Danske Slagterier. - Slg. 2009, I-2168 Rdn. 20 (auch in: EuZW 2009, 384; NVwZ 2009, 771 Rdn. 20); EuGH, Urteil v. 26.1.2010, C-118/o8, Transportes Urbanos. - Slg. 2010, I-635 Rdn. 30; EuGH, Urteil v. 14.3.2013, C-420/11, Leth. - NVwZ 2013, 565 Rdn. 41.

55 Voraussetzung hierfür ist, „dass der Mitgliedstaat die Grenzen, die seinem Ermessen gesetzt sind, offenkundig und erheblich überschritten hat”, so EuGH, Urteil v. 17.4.2007, C-470/03, A.G.M.-COS.MET. - Slg. 2007, I-2749 Rdn. 8o (auch in: EuZW 2007, 480 Rdn. 80; NVwZ 2007, 1282 Rdn. 80); Indikatoren hierfür sind unter anderem „das Maß an Klarheit und Genauigkeit der verletzten Vorschrift, die Frage, ob der Verstoß oder der Schaden vorsätzlich begangen bzw. zugefügt wurde oder nicht, die Entschuldbarkeit oder Unentschuldbarkeit eines etwaigen Rechtsirrtums und der Umstand, dass das Verhalten eines Gemeinschaftsorgans möglicherweise dazu beigetragen hat, dass nationale Maßnahmen oder Praktiken in gemeinschaftsrechtswidriger Weise eingeführt oder aufrechterhalten wurden”, so EuGH, Urteil v. 4.7.200o, C-424/97, Salomone Haim. - Slg. 2000, I-5123 Rdn. 23 (auch in: EuZW 2000, 733 Rdn. 43; NVwZ 2001, 903 Rdn. 43).

56 EuGH, Urteil v. 28.2.2008, C-446/o6. - Slg. 2006, I-11753 Rdn. 210; EuGH, Urteil v. 14.3.2013, C-420/11, Leth. - NVwZ 2013, 565 Rdn. 43. 
Entwicklungsgeschichtlich stellt die richterrechtliche Kreation eines europarechtlichen Staatshaftungsanspruchs eine Reaktion auf die unterbleibende bzw. unzureichende oder verspätete Umsetzung von Richtlinien durch die Mitgliedstaaten dar; der Haftungsanspruch zielt danach auf die Sicherstellung der Wirksamkeit des Unionsrechts. ${ }^{*}{ }^{*} 7$ Außerdem stellt der Gerichtshof mit seiner Rechtsschöpfung eine Kohärenz des Sekundärrechtsschutzes mit der außervertraglichen Haftung der Europäischen Union ${ }^{*} 58$ her. ${ }^{*} 59$ Bemerkenswert sind etliche „punktgenaue” Vorgaben des Gerichtshofs, die aus deutscher Sicht einige Voraussetzungen der Staatshaftung gleichsam außer Kraft setzen:

- Eine Staatshaftung besteht entgegen der deutschen Rechtstradition auch für legislatives Unrecht. ${ }^{*} 60$

- Gehaftet wird ebenfalls für Verstöße der rechtsprechenden Gewalt gegen das Unionsrecht; ${ }^{* 61}$ ein „Richterprivileg”*62 gibt es nicht.

- Verschulden ist bei der Staatshaftung wegen Missachtung des EU-Rechts keine Haftungsvoraussetzung. ${ }^{*} 63$

- Die Ersatzpflicht des Staates hängt nicht von der Verletzung eines bestimmten Rechtsguts (Eigentum) ab. ${ }^{*} 64$

- Zu dem ersatzfähigen Schaden gehört auch der entgangene Gewinn. ${ }^{*} 65$

Die „Europäisierung” weist dem nationalen Staatshaftungsrecht eine primär instrumentelle Funktion zu; es verliert seinen Eigenwert und dient in erster Linie der Durchsetzung der unionsrechtlich begründeten Haftung des Mitgliedstaates im Falle schadensverursachender EU-Rechtsverstöße. ${ }^{* 66}$

\section{Verwaltungsverfahrensrecht}

Zur Europäisierung des Verwaltungsrechts darf das Verwaltungsverfahrensrecht nicht ausgeblendet bleiben. Auch wenn sich allzu pauschale und holzschnittartige Darstellungen verbieten, ${ }^{* 67}$ können ausgewählte Bereiche doch die unterschiedlichen Rationalitäten des deutschen Rechts und des Unionsrechts verdeutlichen.

57 EuGH, Urteil v. 5.3.1996, C-46/93 und C-48/93, Brasserie du Pêcheur. - Slg. 1996, I-1029 Rdn. 39 (auch in: EuZW 1996, 205 Rdn. 39; NJW 1996, 1267 Rdn. 39); A. K. Mangold (Fn. 1), S. 135ff.

58 Art. 340 Abs. 2 AEUV: „Im Bereich der außervertraglichen Haftung ersetzt die Union den durch ihre Organe oder Bediensteten in Ausübung ihrer Amtstätigkeit verursachten Schaden nach den allgemeinen Rechtsgrundsätzen, die den Rechtsordnungen der Mitgliedstaaten gemeinsam sind.”

59 Ausdrücklich betont von EuGH, Urteil v. 5·3.1996, C-46/93 und C-48/93, Brasserie du Pêcheur. - Slg. 1996, I-1029 Rdn. 29 (auch in: EuZW 1996, 205 Rdn. 29; NJW 1996, 1267 Rdn. 29 (Anknüpfung an die außervertragliche Haftung der Gemeinschaft) und Rdn. 33 (einheitliche Anwendung des Gemeinschaftsrechts als Grunderfordernis der Gemeinschaftsrechtsordnung)). Analyse und Kritik zur Rechtsfortbildung seitens des EuGH bei W. Höfling. Vom überkommenen Staatshaftungsrecht zum Recht der staatlichen Einstandspflichten. - W. Hoffmann-Riem, E. Schmidt-Aßmann, A. Voßkuhle (Hrsg.) (Fn. 24), § 51 Rdn. 54ff.

$60 \quad$ EuGH, C-46/93 und C-48/93 (Fn. 59), Rdn. $32 f f$.

$61 \quad$ EuGH, Urteil v. 30.9.2003, C-224/o1, Köbler. - Slg. 2003, I-10239 Rdn. 31 ff. (auch in: EuZW 2003, 718 Rdn. 31ff.; NJW 2003, 3539 Rdn. 31 ff.; NVwZ 2004, 79 Rdn. 31ff.); dazu J. Gundel. Gemeinschaftsrechtliche Haftungsvorgaben für judikatives Unrecht. - Europäisches Wirtschafts- und Steuerrecht (EWS) 2004, S. 8; P. E. Sensburg. Staatshaftung für judikatives Unrecht. - NVwZ 2004, S. 179; F. Kremer. Staatshaftung für Verstöße gegen Gemeinschaftsrecht durch letztinstanzliche Gerichte. - NJW 2004, S. 480; B. W. Wegener, S. Held. Die Haftung der Mitgliedstaaten für die Verletzung von EG-Recht durch nationale Gerichte. - Jura 2004, S. 479; seine Rechtsprechung bestätigend EuGH, Urteil v. 13.6.2006, C-173/03, Traghetti. - Slg. 2006, I-5204 Rdn. 30 ff. (auch in: EuZW 2006, 561 Rdn. 30 ff. (m. Anm. Seegers); NJW 2006, 3337 Rdn. 30 ff.).

$62 \S 839$ Abs. 2 BGB: „Verletzt ein Beamter bei dem Urteil in einer Rechtssache seine Amtspflicht, so ist er für den daraus entstehenden Schaden nur dann verantwortlich, wenn die Pflichtverletzung in einer Straftat besteht. Auf eine pflichtwidrige Verweigerung oder Verzögerung der Ausübung des Amtes findet diese Vorschrift keine Anwendung." Zum Übergang der Haftung auf den Staat durch Art. 34 S. 1 GG vgl. oben Fn. 49.

$63 \quad$ EuGH (Fn. 59), Rdn. 75ff.

64 EuGH, Urteil v. 17.4.2007, C-470/o3, A.G.M.-COS.MET. - Slg. 2007, I-2749 Rdn. 94 (auch in: EuZW 2007, 480 Rdn. 94; NVwZ 2007, 1282 Rdn. 94).

65 EuGH, C-46/93 und C-48/93 (Fn. 59), Rdn. 87; EuGH, C-470/03 (Fn. 64), Rdn. 95.

66 G. Meier. Zur Schadensersatzpflicht der Bundesrepublik Deutschland für Verstöße gegen Gemeinschaftsrecht. - NVwZ 1996, S. 661.

67 W. Kahl. 35 Jahre Verwaltungsverfahrensgesetz - 35 Jahre Europäisierung des Verwaltungsverfahrensrechts. - NVwZ 2011, S. 451. 


\section{Der Verfahrensgedanke im Verwaltungsrecht}

Ausgangspunkt der Überlegungen ist der unterschiedliche Stellenwert, der dem verfahrensrechtlichen Denken in den beiden Rechtsordnungen beigemessen wird. Dabei handelt es sich keineswegs nur um rechtswissenschaftliche Zuschreibungen; entscheidende Prägungen gehen von der Rechtsprechung aus. ${ }^{* 68}$

\section{a) „Dienende Funktion” des Verwaltungsverfahrens im deutschen Recht}

Nach vorherrschendem deutschem Rechtsverständnis ist das Verwaltungsrecht primär materiellrechtlich ausgerichtet. Dem Verwaltungsverfahrensrecht wird demgegenüber eine nachrangige Bedeutung zuerkannt. ${ }^{*} 69$ Ausdrücklich spricht die Rechtsprechung davon, das Verwaltungsverfahren nehme im Verhältnis zum materiellen Verwaltungsrecht eine „dienende Funktion” wahr. ${ }^{* 70}$ Das Verwaltungsverfahren(srecht) fungiert in erster Linie als Verwirklichungsmodus des materiellen Rechts. ${ }^{*}{ }^{71}$ Die Idee der inhaltlichen Richtigkeitsgewähr - auch - durch ein ordnungsgemäß durchgeführtes Verwaltungsverfahren tritt zurück. ${ }^{*} 2$ Das Verfahren ist danach von begrenztem Wert. ${ }^{*} 3$

Dieses Denken findet in Deutschland im positiven Recht einen signifikanten Niederschlag:

- Behördliche Verfahrenshandlungen sind grundsätzlich gerichtlich nicht angreifbar; Rechtsbehelfe gegen behördliche Verfahrenshandlungen können nur gleichzeitig mit den gegen die Sachentscheidung zulässigen Rechtsbehelfen geltend gemacht werden, es sei denn, behördliche Verfahrenshandlungen können vollstreckt werden oder ergehen gegen einen Nichtbeteiligten. ${ }^{*} 74$

- Verfahrensfehler, die nicht zur Nichtigkeit der Verwaltungsentscheidung führen, können geheilt werden; die dafür notwendigen Handlungen kann die Behörde auch noch während eines verwaltungsgerichtlichen Verfahrens bis zum Abschluss der letzten Tatsacheninstanz nachholen. ${ }^{*} 75$

- Eine Verletzung von Verfahrensvorschriften ist im Ergebnis unbeachtlich, wenn offensichtlich ist, dass der Rechtsverstoß die Entscheidung in der Sache nicht beeinflusst hat. ${ }^{*} 76$

Diese drei Beispiele aus dem positiven Recht verdeutlichen, dass das deutsche Verwaltungsrecht die Ergebnisrichtigkeit in der Sache anstrebt und der Verfahrensrichtigkeit in diesem Zusammenhang nur eine untergeordnete Rolle zuweist.

68 Vgl. die Rechtsprechungsanalyse von J. Kersten. Das Verwaltungsverfahrensgesetz im Spiegel der Rechtsprechung der Jahre 2004-2012. - Die Verwaltung (46) 2013, S. 87ff. - DOI: http://dx.doi.org/10.379o/verw. 1.8746.

69 R. Wahl. Das Verhältnis von Verwaltungsverfahren und Verwaltungsprozessrecht in europäischer Sicht. - DVBl. 2003, S. $1287 f$.; K.-P. Dolde. Verwaltungsverfahren und Deregulierung. - NVwZ 2006, S. 858.

70 BVerwG, Urteil v. 15.1.1982, 4 C 26/78. - BVerwGE 64, 325 (333f.); BVerwG, Urteil v. 29.4.1993, 7 A 2/92. - BVerwGE 92, 258 (263); BVerwG, Urteil v. 10.10.2002, 6 C 8/01. - BVerwGE 117, 93 (104); BVerwG, Urteil v. 24.11.2011, 9 A 23/10. BVerwGE 141, 171 (173); vgl. ferner M. Burgi. Die dienende Funktion des Verwaltungsverfahrens: Zweckbestimmung und Fehlerfolgenrecht in der Reform. - DVBl. 2011, S. 1317ff.

71 R. Wahl. Verwaltungsverfahren zwischen Verwaltungseffizienz und Rechtsschutzauftrag. - Veröffentlichungen der Vereinigung der Deutschen Staatsrechtslehrer (VVDStRL) (41) 1983, S. $153 \mathrm{ff}$.

72 Demgegenüber „dienende Funktion” und „Eigenwert” des Verwaltungsverfahrens in ein ausgewogenes Zuordnungsverhältnis setzend E. Gurlit, M. Fehling. Der Eigenwert des Verfahrens im Verwaltungsrecht. - VVDStRL (70) 2011, S. 227ff. und S. $278 f f$.

73 J. Pietzcker. Wie weit trägt der Verfahrensgedanke? - Europäisches Recht zwischen Bewährung und Wandel: Festschrift für Dieter H. Scheuing. Baden-Baden: Nomos 2011, S. 374ff.

$74 \S 44 a$ VwGO; dazu BVerwG, Urteil v. 10.2.1999, 11 A 21-98. - NJW 1999, 1729 und BVerwG, Urteil v. 30.1.2002, 9 A 20.01. DVBl. 2002, 1118; Bayerischer Verwaltungsgerichtshof, Beschluss v. 4.12.2012, 7 ZB 12.1816. - BayVBl. 2013, 308; zu einem Ausnahmefall BVerwG, Urteil v. 1.9.2009, 6 C 4/o9. - BVerwGE 134, 368 (auch in: NVwZ 2009, 1558).

$75 \S 45$ VwVfG; zur Bauleitplanung vgl. § 214 Abs. 4 Baugesetzbuch.

76 § 46 VwVfG; zur Bauleitplanung vgl. § 214 Abs. 1 Baugesetzbuch. 


\section{b) Eigenwert des Verwaltungsverfahrens im Unionsrecht}

In europarechtlicher Perspektive ist dem Verwaltungsverfahren(srecht) eine eigenständige Funktion zugewiesen. Gleichberechtigt werden zwei Funktionen erfüllt; das Verfahren dient der Verwaltung als Instrument der Rechtsverwirklichung und zugleich als Schutz der Rechte des Einzelnen, es hat demnach auch einen Eigenwert. ${ }^{*} 77$

Das bedeutet nicht, dass dem Unionsverwaltungsrecht die Heilung oder die Unbeachtlichkeit von Verfahrensfehlern prinzipiell fremd wäre. ${ }^{*} 78$ Aber die Voraussetzungen sind andere als im deutschen Verwaltungsrecht. So kommt z. B. die Fehlerheilung nach Abschluss des Verwaltungsverfahrens grundsätzlich nicht in Betracht. ${ }^{* 79}$ Dem liegt die Erwägung zu Grunde, dass nach Beginn des gerichtlichen Verfahrens nicht mehr alle Optionen im Sinne einer realen Fehlerheilung seitens der Verwaltung bestehen. ${ }^{*} 0$ Sogar im zweitinstanzlichen Verwaltungsverfahren macht der Gerichtshof die Heilung eines Verfahrensfehlers davon abhängig, dass alle Optionen offen sind und die Heilung in diesem Verfahrensstadium noch eine im Hinblick auf den Ausgang des Verfahrens effektive Nachholung der unterbliebenen Verfahrenshandlung ermöglicht. ${ }^{*} 81$ Bei Sachverhalten mit Bezug zum Unionsrecht bedarf das deutsche Recht (§ 45 Abs. 2 VwVfG) danach einer partiellen Korrektur. ${ }^{*} 2$

\section{Verfahrensrechtliche Stellung des Einzelnen}

\section{a) Abhängigkeit des deutschen Verfahrensrechts vom materiellen Recht}

Die „dienende Funktion” des Verwaltungsverfahrens bleibt nach deutschem Verständnis nicht ohne Konsequenzen für die Rechtsstellung des Einzelnen. Da das Verwaltungsverfahrensrecht auf die Hervorbringung rechtmäßiger (und zweckmäßiger) Verwaltungsentscheidungen ausgerichtet ist, ${ }^{*} 3$ begründen individuelle Verfahrensrechte in Bezug auf die Sachentscheidung der Verwaltung keinen subjektiven Schutz. Wendet sich ein von der Sachentscheidung Betroffener mit Verfahrensrügen gegen die Verwaltungsentscheidung, ist er nur dann rechtlich geschützt, wenn sich der Verfahrensrechtsverstoß auf die materiellrechtliche Position des Betroffenen ausgewirkt hat. ${ }^{*} 4$

\section{b) Selbstständige Verfahrensrechte im Unionsrecht}

Das Unionsrecht statuiert demgegenüber selbstständige Verfahrenspositionen. Das Verwaltungsverfahrensrecht dient nicht nur der Verwirklichung des materiellen Rechts, es bietet auch eine Gewähr für die Richtigkeit der Verwaltungsentscheidung. ${ }^{*} 5$ Dieser Ansatz kann Konsequenzen für die selbstständige Durchsetzung des Verwaltungsverfahrensrechts haben. Das bekannteste Beispiel hierfür ist das Einklagen der Umweltverträglichkeitsprüfung bei bestimmten (Groß-)Projekten. ${ }^{* 86}$ Vereinzelt sind deutsche Gerichte diesem Ansatz

77 W. Kahl. Grundrechtsschutz durch Verfahren in Deutschland und in der EU. - Verwaltungsarchiv 2004, S. 8 ff.; M. Burgi. Verwaltungsverfahrensrecht zwischen europäischem Umsetzungsdruck und nationalem Gestaltungsunwillen. - JZ 2010, S. 105ff.; T. v. Danwitz (Fn. 14), S. 530.

78 J. Schwarze. Die Rechtsprechung des EuGH zur Relevanz von Fehlern im Verwaltungsverfahren. - D. Heckmann, R. P. Schenke, G. Sydow (Hrsg.). Verfassungsstaatlichkeit im Wandel: Festschrift für Thomas Würtenberger zum 70. Geburtstag. Berlin: Duncker \& Humblot 2013, S. $1203 \mathrm{ff}$.

79 EuGH, Urteil v. 24.10.1996, C-329/93 u.a. - Slg. 1996, I-5151 Rdn. 48 (auch in: NVwZ 1997, 475 Rdn. 48 (Begründungsmangel im Gerichtsverfahren nicht mehr heilbar)); ebenso zu einem Anhörungsmangel EuG, Urteil v. 9.11.1995, T-346/94, France-aviation. - Slg. 1995, II-2843 Rdn. 39 (auch in: EuZW 1996, 602 Rdn. 39); ferner B. Bredemeier. Kommunikative Verfahrenshandlungen im deutschen und europäischen Verwaltungsrecht. Tübingen: Mohr Siebeck 2007, S. 414ff.

80 J. Kokott, C. Sobotta. Das BVerwG und das europäische Umweltrecht. - NVwZ Beilage 2013/1, S. 51.

81 EuGH, Urteil v. 15.1.2013, C-416/10. - NuR 2013, 113 Rdn. $89 f$.

82 W. Kahl (Fn. 67), S. 451f.

83 BVerfG, Beschluss v. 20.2.2002, 1 BvL 19/97 u.a. - BVerfGE 105, 48 (60) (auch in: NVwZ 2002, 1101 (1102)).

84 BVerwG, Urteil v. 25.01.1996, 4 C 5/95. - BVerwGE 100, 238 (251f.); BVerwG, Urteil v. 20.12.2011, 9 A 30/10. - NVwZ 2012, 573 Rdn. 19.

85 VGH Baden-Württemberg, Urteil v. 29.06.2006, 11 S 2299/05. - Verwaltungsblätter für Baden-Württemberg 2007, 109 (114).

86 EuGH, Urteil v. 7.1.2004, C-201/o2, Wells. - Slg. 2004, I-723 Rdn. 56ff. (auch in: NVwZ 2004, 593 Rdn. 56ff.; DVBl. 2004, 370 (m. Anm. Kerkmann, DVBl. 2004, 1287) Rdn. 56 ff.); bestätigt durch EuGH, Urteil v. 21.3.2013, C-244/12, Salzburger Flughafen. - NVwZ 2013, 707 Rdn. 46. 
gefolgt. ${ }^{*}{ }^{87}$ Nach wie vor handelt es sich dabei aber um eine seltene Ausnahme vom überkommenen deutschen Konzept. Hier besteht Potential für die Europäisierung des deutschen Verwaltungsverfahrensrechts.

\section{Verfahrensfehlerfolgen}

Tritt keine Heilung eines Verfahrensfehlers ein, stellt sich die Frage nach den Fehlerfolgen. Allgemein*88 entspricht es europäischen Standards, dass ein Verfahrensrechtsverstoß für unbeachtlich erklärt werden kann, wenn auszuschließen ist, dass sich der Fehler auf die behördliche Sachentscheidung ausgewirkt hat. ${ }^{*} 9$ Entscheidend sind die Voraussetzungen für den Eintritt dieser Rechtsfolge.

\section{a) Nachweis der konkreten Kausalität im deutschen Recht}

Für den rechtlich relevanten Einfluss eines Verfahrensfehlers auf die Sachentscheidung ist nach deutschem Allgemeinen Verwaltungsrecht ein Kausalzusammenhang erforderlich ( $§ 46 \mathrm{VwVfG}^{*}{ }^{\circ}{ }^{\circ}$ ). Besondere Bedeutung hat die Thematik im Umwelt- und Planungsrecht erlangt. Von der Rechtsprechung wird der Kausalzusammenhang bejaht, wenn nach den Umständen des Einzelfalls die konkrete Möglichkeit besteht, dass z.B. eine angegriffene Planungsentscheidung (ein Planfeststellungsbeschluss) ohne den Verfahrensfehler für den Betroffenen (den Kläger) günstiger ausgefallen wäre. ${ }^{* 1}$ Wird der Kausalzusammenhang verneint, bleibt der Verfahrensfehler - falls nicht (ausnahmsweise) ein absolutes Verfahrensrecht ${ }^{*}{ }^{92}$ verletzt worden ist - rechtlich folgenlos.

Bei rechtsdogmatischer Beurteilung dieser Konzeption kann ein Verfahrensfehler immer dann Einfluss auf die Sachentscheidung nehmen, wenn die Behörde über Entscheidungsspielräume verfügt (Ermessen, Beurteilungsspielraum, planerische Gestaltung). Muss dagegen auf der Grundlage einer gesetzlich gebundenen Verwaltungsentscheidung angenommen werden, auch ohne den Verfahrensfehler wäre in der Sache nicht anders entschieden worden, bleibt der Verfahrensrechtsverstoß unbeachtlich. In Deutschland wird dieses Ergebnis nun allerdings auch bei der Existenz behördlicher Entscheidungsspielräume erzielt, ${ }^{*} 93$ weil nach der Judikatur der Betroffene (Kläger) nachweisen muss, dass ohne den Verfahrensfehler die behördliche Sachentscheidung anders ausgefallen wäre. ${ }^{*} 4$ Dieser Nachweis gelingt in der Praxis nur selten. ${ }^{*} 95$

\section{b) Erheblichkeit wesentlicher Verfahrensfehler nach Unionsrecht}

Findet ein individuelles Recht im Verwaltungsverfahren seine Begründung im EU-Recht, läuft jenes Recht nach der deutschen Konzeption weitgehend leer, was mit dem unionsrechtlichen Effektivitätsgebot kaum vereinbar ist. Der im Schrifttum unterbreitete Vorschlag, dem Schutz Betroffener dienende wesentliche Verfahrensrechtspositionen des Unionsrechts als nicht relativierbare „absolute Rechte”, deren Verletzung

87 Oberverwaltungsgericht Rheinland-Pfalz, Beschluss v. 25.1.2005, 7 E 12117/04. - NVwZ 2005, 1208; ablehnend dazu H. Lecheler. Isolierte Anfechtung von Verfahrensfehlern ohne materielle Beschwer kraft Europarechts? - NVwZ 2005, S. 1156f .

88 Zur spezialgesetzlichen Fehlerfolgenregelung im Umwelt-Rechtsbehelfsgesetz F. Schoch. - W. Hoffmann-Riem, E. SchmidtAßmann, A. Voßkuhle (Hrsg.) (Fn. 24), § 50 Rdn. 314ff.

89 E. Gurlit, M. Fehling (Fn. 72), S. $293 f f$.

90 Die Vorschrift lautet: „Die Aufhebung eines Verwaltungsaktes, der nicht nach § 44 nichtig ist, kann nicht allein deshalb beansprucht werden, weil er unter Verletzung von Vorschriften über das Verfahren, die Form oder die örtliche Zuständigkeit zustande gekommen ist, wenn offensichtlich ist, dass die Verletzung die Entscheidung in der Sache nicht beeinflusst hat."

$91 \quad$ BVerwG, Urteil v. 29.10.2008, 6 C 38/07. - NVwZ 2009, 653 Rdn. 42; BVerwG, Urteil v. 14.4.2010, 9 A 5/o8. - BVerwGE 136, 291 Rdn. 25 (auch in: NVwZ 2010, 1225 Rdn. 25); BVerwG, Urteil v. 24.3.2011, 7 A 3/10. - NVwZ 2011, 1124 Rdn. 84; BVerwG, Urteil v. 24.11.2011, 9 A 23/10. - BVerwGE 141, 171 Rdn. 15 (auch in: NVwZ 2012, 557 Rdn. 15); BVerwG, Urteil v. 31.7.2012, 4 A 7001/11 u.a. - BVerwGE 144, 44 Rdn. 34 (auch in: NVwZ 2013, 297 Rdn. 34).

92 Einzelheiten zu dieser Kategorie von Verfahrensrechten bei F. Schoch. - W. Hoffmann-Riem, E. Schmidt-Aßmann, A. Voßkuhle (Hrsg.) (Fn. 24), § 50 Rdn. 304 (mit zahlreichen Beispielen aus der Praxis).

93 Vgl. z.B. BVerwG, Urteil v. 14.4.2010, 9 A 5/o8. - BVerwGE 136, 291 Rdn. 25 (auch in: NVwZ 2010, 1225 Rdn. 25); BVerwG, Urteil v. 24.3.2011, 7 A 3/10. - NVwZ 2011, 1124 Rdn. 86; BVerwG, Urteil v. 24.11.2011, 9 A 23/10. - BVerwGE 141, 171 Rdn. 18 (auch in: NVwZ 2012, 557 Rdn. 18); BVerwG, Urteil v. 31.7.2012, 4 A 7001/11 u.a. - BVerwGE 144, 44 Rdn. 35ff. und Rdn. 46 (auch in: NVwZ 2013, 297 Rdn. 35ff. und Rdn. 46).

94 BVerwG, Urteil v. 29.10.2008, 6 C 38/o7. - NVwZ 2009, 653 Rdn. 42; BVerwG, Urteil v. 24.11.2011, 9 A 23/10. - BVerwGE 141, 171 Rdn. 18 (auch in: NVwZ 2012, 557 Rdn. 18).

95 B. Bredemeier (Fn. 79), S. 369, fordert, die Darlegungs- und Beweislast der Behörde aufzuerlegen. 
immer beachtlich ist, zu deuten, ${ }^{*}{ }^{6}$ wird von der Rechtsprechung unter Hinweis auf die Verfahrensautonomie der Mitgliedstaaten abgelehnt. ${ }^{*}{ }^{7}$ Am Beispiel der Umweltverträglichkeitsprüfung (UVP) zeigt sich jedoch, dass die deutsche Judikatur kaum durchzuhalten ist. Denn individuelle Verfahrensrechte nach der UVP-Richtlinie ${ }^{*} 98$ verlangen eine effektive Durchsetzung im innerstaatlichen Recht. ${ }^{*} 99$

In einem Vertragsverletzungsverfahren gegen die Bundesrepublik Deutschland hat die EU-Kommission in ihrer mit Gründen versehenen Stellungnahme die Praxis der deutschen Gerichte zur Relativierung des Verwaltungsverfahrensrechts gerügt: Werde die Beweislast für die Kausalität eines Verfahrensfehlers in Bezug auf die behördliche Sachentscheidung dem Betroffenen (Kläger) auferlegt, stelle dies eine Verletzung des Rechts auf einen wirksamen Zugang zu einer gerichtlichen Überprüfung von behördlichen UVP-Entscheidungen dar. Bei einem erheblichen Verfahrensfehler (z.B. Verstoß gegen Informations- und Beteiligungsrechte der Öffentlichkeit) müsse das nationale Recht die Aufhebung der Verwaltungsentscheidung vorsehen, ohne dass der Betroffene (Kläger) einen Kausalzusammenhang mit dem Ergebnis der Verwaltungsentscheidung zu beweisen habe. ${ }^{*} 100$

Eine rechtliche Klärung in diesem Punkt kann auch ein vom Bundesverwaltungsgericht in Gang gesetztes Vorabentscheidungsverfahren ${ }^{* 101}$ bringen. In seinem Schlussantrag betont der Generalanwalt, dass bei der Anwendung des Kausalitätskriteriums (im Rahmen des § 46 VwVfG) das Effektivitätsprinzip zu beachten sei; für besonders wichtige Verfahrensvorschriften (nach der UVP-Richtlinie) müsse auf das Erfordernis der Kausalität für das Ergebnis des Verwaltungsverfahrens (d.h. die behördliche Sachentscheidung) vollständig verzichtet werden. ${ }^{*}{ }^{102}$ Der Gerichtshof hat nun entscheiden, dass eine zwar durchgeführte, aber mit erheblichen Fehlern versehene Umweltverträglichkeitsprüfung vom innerstaatlichen Verwaltungsverfahrensrecht nicht generell sanktionslos gestellt werden kann; ein solcher Automatismus ist unionsrechtswidrig. ${ }^{*} 103$

\section{Verwaltungsprozessrecht}

In einem dritten Untersuchungsfeld soll, wenn auch nur kurz, auf die Europäisierung im Verwaltungsprozessrecht eingegangen werden. ${ }^{*} 104$ Zur Illustration des Befundes werden drei Komplexe herangezogen, die beispielhaft die besonders intensive Einwirkung des Unionsrechts auf das gerichtliche Verfahrensrecht veranschaulichen.

\section{Klagebefugnis}

Der individuelle Zugang zum verwaltungsgerichtlichen Rechtsschutz und damit zur Kontrolle der Verwaltung durch die Judikatur wird in erster Linie durch die Klagebefugnis markiert. Dazu kennt das deutsche Verwaltungsprozessrecht die individuelle Klageberechtigung und den entsubjektivierten Verwaltungsrechtsschutz in Gestalt der Verbandsklage (§ 42 Abs. 2 VwGO ${ }^{* 105}$ ).

96 J. Held. Individualrechtsschutz bei fehlerhaftem Verwaltungsverfahren. - NVwZ 2012, S. 467.

97 BVerwG, Urteil v. 29.10.2008, 6 C 38/o7. - NVwZ 2009, 653 Rdn. 41.

98 Richtlinie 2011/92/EU des Europäischen Parlaments und des Rates vom 13.12.2011 über die Umweltverträglichkeitsprüfung bei bestimmten öffentlichen und privaten Projekten. - ABl. Nr. L 26/1.

99 EuGH, Urteil v. 7.1.2004, C-201/o2, Wells. - Slg. 2004, 723 Rdn. 69 (auch in: NVwZ 2004, 593 Rdn. 69): Rücknahme oder Aussetzung der Verwaltungsentscheidung zwecks Durchführung der UVP.

100 Europäische Kommission, Vertragsverletzung Nr. 2007/4267, Stellungnahme vom 26.4.2013 C(2013) 2173 final, S. $12 f$.

101 BVerwG, Beschluss v. 10.1.2012, 7 C 20/11, Gemeinde Altrip. - NVwZ 2012, 448.

102 GA Cruz Villalón, Schlussanträge v. 20.6.2013, C-72/12, Rdn. 106.

103 EuGH, Urteil v. 7.11.2013, C-72/12, Gemeinde Altrip. - NVwZ 2014, 49 Rdn. 47ff.; dazu M. Böhm. Die gerichtliche Kontrolle von UVP-Fehlern. - Umwelt- und Planungsrecht (UPR) 2014, S. 201; T. Bunge, Rechtsfolge von Verfahrensfehlern bei der Umweltverträglichkeitsprüfung. - Natur und Recht (NuR) 2014, S. 305; T. Siegel, Ausweitung und Eingrenzung der Klagerechte im Umweltrecht. - NVwZ 2014, S. 973.

104 Ausführlich dazu D. Ehlers. Die Europäisierung des Verwaltungsprozessrechts. Köln: Heymanns 1999; F. Schoch. Die Europäisierung des verwaltungsgerichtlichen Rechtsschutzes. Berlin: de Gruyter 2000; O. Dörr. Der europäisierte Rechtsschutzauftrag deutscher Gerichte. Tübingen: Mohr Siebeck 2003; T. Dünchheim. Verwaltungsprozessrecht unter europäischem Einfluss. Berlin: Duncker \& Humblot 2003. Im Überblick F. Schoch. Die Europäisierung des Verwaltungsprozessrechts. E. Schmidt-Aßmann (Hrsg.). Festgabe 50 Jahre Bundesverwaltungsgericht. Köln: Heymanns 2003, S. 507 ff.; D. Ehlers. Die Europäisierung des Verwaltungsprozessrechts. - DVBl. 2004, S. 1441ff.; C. Steinbeiß-Winkelmann. Europäisierung des Verwaltungsrechtsschutzes. - NJW 2010, S. 1233ff.

105 Die Vorschrift lautet: „Soweit gesetzlich nichts anderes bestimmt ist, ist die Klage nur zulässig, wenn der Kläger geltend macht, durch den Verwaltungsakt oder seine Ablehnung oder Unterlassung in seinen Rechten verletzt zu sein.” 


\section{a) Individuelle Klageberechtigung}

Der Individualrechtsschutz erfolgt in Deutschland nach Maßgabe des subjektiven öffentlichen Rechts. Das Verwaltungsprozessrecht knüpft in diesem Punkt an das Allgemeine Verwaltungsrecht an. ${ }^{*}{ }^{106}$ Es gilt die Schutznormtheorie. Anerkannt ist weder die Popularklage noch die Interessentenklage. Klagebefugt ist im Verwaltungsprozess nur, wer geltend machen kann, durch die von ihm angefochtene staatliche Maßnahme bzw. durch die behördliche Ablehnung oder Unterlassung der beantragten Maßnahme in eigenen Rechten verletzt zu sein. ${ }^{*} 107$

In diesem System findet die skizzierte funktionale Subjektivierung nach Maßgabe des Unionsrechts ${ }^{* 108}$ ihren vollständigen Niederschlag im innerstaatlichen deutschen Verwaltungsprozessrecht: Individuelle Klagerechte werden anerkannt, soweit eine Rechtsnorm auf die Verleihung von Rechten an den Einzelnen zielt. Dabei werden, wie gesehen, aggregierte Interessen Privater nicht entsubjektiviert, sie bleiben individuell einklagbar. Klageberechtigt ist jede Person, die Träger des personalen Schutzguts ist und durch das mit der Klage bekämpfte behördliche Verhalten nachteilig in seinen rechtlich geschützten Interessen beeinträchtigt worden ist. ${ }^{*} 109$ Das Unionsrecht anerkennt also die Schutznormtheorie, allerdings in Gestalt der funktionalen Subjektivierung von Rechtsnormen mit personalen Schutzgütern (z.B. Schutz der Verbraucher, Schutz der menschlichen Gesundheit).

\section{b) Altruistische Verbandsklage}

Die altruistische Verbandsklage ist dem Grunde nach prozessrechtlich unbedenklich (§ 42 Abs. 2 Alt. 1 VwGO). Große praktische Bedeutung hat sie im Umweltrecht und steht dort im Dienste einer objektiven Rechtskontrolle der Verwaltung. Maßgebend sind europarechtliche Vorgaben, ${ }^{*}{ }^{* 10}$ die ihrerseits Völkerrecht - in Gestalt der Aarhus-Konvention (AK) ${ }^{*} 111$ - umsetzen. Ausdrücklich hat der Europäische Gerichtshof betont, nach dem einschlägigen Unionsrecht müssten klageberechtigte Verbände die Verletzung von Vorschriften des Umweltschutzrechts auch dann gerichtlich geltend machen können, wenn derartige Vorschriften nur die Allgemeinheit schützen und nicht auch Rechte bzw. Rechtsgüter des Individuums. ${ }^{*}{ }_{112}$ Diese gegen das frühere deutsche Umweltrechtsbehelfsgesetz ${ }^{* 13}$ gerichtete Entscheidung hat Anfang 2013 zu einer Reaktion des deutschen Gesetzgebers geführt. ${ }^{*} 14$ Schon ist in der Rechtsprechung akzeptiert worden, dass ein klageberechtigter Umweltverband einen Verstoß gegen umweltschützende Rechtsvorschriften gerichtlich geltend machen kann, auch wenn diese nationalen Vorschriften keinen subjektiven Rechtsschutz Einzelner gewähren. ${ }^{*} 15$

Festzuhalten bleibt, dass die Klagebefugnis ganz wesentliche Impulse durch das EU-Recht erhält. ${ }^{* 16}$ Es ist an der Zeit, dass in Deutschland die maßgebenden Organe der Gesetzgebung und der Rechtsprechung

106 Vgl. dazu oben II. 1.

107 Vgl. etwa J. Ruthig. Subjektiver Rechtsschutz und objektive Rechtskontrolle in Deutschland. - W.-R. Schenke, J. Hyun Seok (Hrsg.). Rechtsschutz gegen staatliche Hoheitsakte in Deutschland und Korea. Berlin: Duncker \& Humblot 2006, S. 117ff.; zu den Rechtsproblemen bei Drittklagen H. Geiger. Die Konkurrentenklage im Verwaltungsprozessrecht. - BayVBl. 2010, S. $517 f f$.

108 Einzelheiten dazu oben II. 1. b); ausführlich F. Schoch. - W. Hoffmann-Riem, E. Schmidt-Aßmann, A. Voßkuhle (Hrsg.) (Fn. 24), § 50 Rdn. 149ff.

109 A. Epiney. Primär- und Sekundärrechtsschutz im Öffentlichen Recht. - VVDStRL (61) 2002, S. 405 f; O. Dörr, C. Lenz. Europäischer Verwaltungsrechtsschutz. Baden-Baden: Nomos 2006, Rdn. 433; T. v. Danwitz (Fn. 14 ), S. 514.

110 Art. 25 RL 2010/75/EU des Europäischen Parlaments und des Rates vom 24.11.2010 über Industrieemissionen (integrierte Vermeidung und Verminderung der Umweltverschmutzung). - ABl. Nr. L 334/17; Art. 11 RL 2011/92/EU vom 13.12.2011 über die Umweltverträglichkeitsprüfung bei bestimmten öffentlichen und privaten Projekten. - ABl. Nr. L 26/1.

111 UN/ECE-Konvention über den Zugang zu Informationen, die Öffentlichkeitsbeteiligung an Entscheidungsverfahren und den Zugang zu Gerichten in Umweltangelegenheiten („Aarhus-Konvention”), in Deutschland umgesetzt durch Gesetz vom 9.12.2006 (BGBl. II S. 1251) und - zum Ersten Aarhus-Änderungs-Übereinkommen - Gesetz vom 17.7.2009 (BGBl. II S. 794).

112 EuGH, Urteil v. 12.5.2011, C-115/o9, Trianel. - Slg. 2011, I-3701 Rdn. 37ff. (auch in: EuZW 2011, 510 (m. Anm. Hellriegel); NVwZ 2011, 801 (m. Anm. Schlacke); DVBl 2011, 757 (m. Anm. Durner/Paus)).

113 Umwelt-Rechtsbehelfsgesetz vom 7.12.2006. - BGBl. I S. 2816.

114 Gesetz zur Änderung des Umwelt-Rechtsbehelfsgesetzes vom 21.1.2013. - BGBl. I S. 95.

115 Oberverwaltungsgericht Rheinland-Pfalz, Beschluss v. 31.1.2013, 1 B 11201/12. - NVwZ 2013, 883 (884).

116 W. Porsch. Verwaltungsgerichtlicher Rechtsschutz im Umweltrecht. - NVwZ 2013, S. 1393; K. F. Gärditz, Verwaltungsgerichtlicher Rechtsschutz im Umweltrecht. - NVwZ 2014, S. 1. 
ihre Abneigung gegen die breit angelegte Verbandsklage im Umweltrecht ablegen und sich gegenüber den europarechtlichen Entwicklungen im Interesse der Sache, also des Umweltschutzes, aufgeschlossen zeigen.

Das Bundesverwaltungsgericht hat nun reagiert und einem Umweltverband die Klagebefugnis gegen die Ablehnung der Aufstellung eines Luftreinhalteplans zugesprochen. Grundlage dafür sei allerdings nicht die Verbrauchsklagebefugnis ( $\$ 42$ Abs. 2 Alt. 1 VwGO), sondern die Geltendmachung der Verletzung eigener Rechte (§ 42 Abs. 2 Alt. 2 VwGO). Zur Sicherung der praktischen Wirksamkeit des Unions(umwelt) rechts wird dem Verband eine „prokuratorische” Rechtsstellung zugewiesen. ${ }^{*}{ }^{* 17}$ Der Umweltverband handelt danach als „Treuhänder” zur Durchsetzung des EU-Rechts. Methodisch ist die Entscheidung sicherlich angreifbar. In der Sache belegt sie aber die fortschreitende Europäisierung des nationalen Rechts.

\section{Subjektiver Rechtswidrigkeitszusammenhang}

\section{a) Begründetheit der Klage: eigene Rechtsverletzung}

In einem engen Zusammenhang mit dem deutschen Konzept zur Klagebefugnis steht der subjektive Rechtswidrigkeitszusammenhang. Er findet Niederschlag im positiven Recht ${ }^{* 118}$ und besagt, dass eine zulässige Klage nicht schon bei objektiver Rechtswidrigkeit eines Verwaltungsakts begründet ist, sondern zusätzlich die dadurch erfolgende subjektive Rechtsverletzung des Klägers voraussetzt. ${ }^{*} 19$ Das bedeutet, dass trotz der Rechtswidrigkeit eines Verwaltungsakts die Klage unbegründet ist, wenn der Kläger nicht zugleich in eigenen Rechten verletzt ist. ${ }^{*} 120$

Ausnahmen von diesem Konzept gibt es nur wenige. Das bekannteste Beispiel, das auch in der Praxis von Bedeutung ist, ist die Klage gegen einen Planfeststellungsbeschluss mit enteignungsrechtlicher Verwirkung. Betroffene Grundeigentümer können eine umfassende gerichtliche Überprüfung der behördlichen Planungsentscheidung (z.B. für einen Straßenbau) verlangen. ${ }^{* 121}$ Die nicht enteignungsrechtlich betroffenen Kläger können keine insgesamt fehlerfreie behördliche Planungsentscheidung beanspruchen; relevant sind nur die Verletzung gerade diese Kläger schützender Normen sowie eine nicht ordnungsgemäße Abwägung ihrer geschützten privaten Belange. ${ }^{* 122}$

\section{b) Europarechtliche Risiken}

Bislang ist dieses deutsche Konzept europarechtlich ohne Beanstandung geblieben. Das gilt sogar - modifiziert - für die Verbandsklage im Umweltrecht. Ein Umweltverband kann mit Erfolg nur den Verstoß gegen umweltschützende Rechtsvorschriften rügen. ${ }^{*}{ }^{123}$ Eine umfassende, über umweltrechtliche Belange hinausgehende gerichtliche Kontrolle der Verwaltungsentscheidung, also z.B. des Planfeststellungsbeschlusses, findet nicht statt. ${ }^{*} 124$

In einem Vertragsverletzungsverfahren gegen die Bundesrepublik Deutschland wegen des Vorwurfs einer Verletzung der Industrieemissionsrichtlinie und der UVP-Richtlinie ${ }^{*_{125}}$ durch das deutsche

117 BVerwG, Urteil v.5.9.2013, C 21/12. - NVwZ 2014, 64 Rdn. 38 ff.; dazu C. Franzius. Möglichkeiten und Grenzen der richterlichen Rechtsfortbildung zur Bestimmung der Klagebefugnis im Umweltrecht. DVBl. 2014, S. 543; E. Gassner. Zur gerichtlichen Geltendmachung von EU-Rechtsvorschriften, die dem Umweltschutz dienen. - DVBl. 2014, S. 551; M. Lau. Das Urteil des BVerwG vom 5.9.2013. - NVwZ 2014. S. 637.

$118 \S 113$ Abs. 1 S. 1 VwGO bestimmt für die Anfechtungsklage: „Soweit der Verwaltungsakt rechtswidrig und der Kläger dadurch in seinen Rechten verletzt ist, hebt das Gericht den Verwaltungsakt und den etwaigen Widerspruchsbescheid auf.” § 113 Abs. 5 S. 1 VwGO bestimmt für die Verpflichtungsklage (Leistungsklage): „Soweit die Ablehnung oder Unterlassung des Verwaltungsakts rechtswidrig und der Kläger dadurch in seinen Rechten verletzt ist, spricht das Gericht die Verpflichtung der Verwaltungsbehörde aus, die beantragte Amtshandlung vorzunehmen, wenn die Sache spruchreif ist."

119 Das Konzept erläuternd M. Gerhardt. - F. Schoch, J.-P. Schneider, W. Bier (Hrsg.) (Fn. 12), § 113 Rdn. 11ff.

120 Beispiel aus der Praxis: BVerwG, Urteil v. 26.1.2011, 6 C 2/10. - NVwZ 2011, 613 (Anfechtungsklage eines Dritten gegen Bescheid der Regulierungsbehörde zur Frequenzzuteilung im Telekommunikationsrecht).

121 Zuletzt in diesem Sinne (mit umfangreichen weiteren Nachweisen) BVerwG, Urteil v. 14.11.2012, 9 C 14/11. - NVwZ 2013, 803 Rdn. 10.

122 BVerwG, Urteil v. 10.10.2012, 9 A 20/11. - NVwZ 2013, 645 Rdn. 11; BVerwG, Urteil v. 10.10.2012, 9 A 19/11. - NVwZ 2013, 649 Rdn. 14 (jeweils m.w.N.).

123 BVerwG, Urteil v. 10.10. 2012, 9 A 18/11. - NVwZ 2013, 642.

124 Ibid., Rdn. 18; VGH Baden-Württemberg, Urteil v. 20.07.2011, 10 S 2102/o9. - Beck-Rechtsprechung 2011, 54899.

125 Nachweise dazu oben Fn. 110. 
Verwaltungsprozessrecht greift die EU-Kommission nun den subjektiven Rechtswidrigkeitszusammenhang an: Sei die Klage einer natürlichen oder juristischen Person zulässig, dürfe die gerichtliche Prüfung nicht auf solche Vorschriften beschränkt werden, die Rechte Einzelner begründen; vielmehr müsse die Einhaltung aller Verfahrensbestimmungen und materiellrechtlichen Vorschriften kontrolliert werden. ${ }^{*}{ }^{126}$ Zur Begründung beruft sich die EU-Kommission auf das „Trianel”-Urteil des Gerichtshofs; die von der EU-Kommission in Bezug genommene Aussage dieser Entscheidung ${ }^{* 127}$ befasst sich allerdings nur mit der Zulässigkeit einer umweltrechtlichen Verbandsklage unter dem Aspekt des Rügepotentials, d.h. zulässiger Klagegründe. Daraus lässt sich jedoch kaum die Pflicht deutscher Gerichte zu einer umfassenden RechtmäBigkeitskontrolle des Verwaltungshandelns ableiten. ${ }^{*} 128$

\section{Vorläufiger Rechtsschutz}

Für das Verwaltungsprozessrecht hat die Europäische Union keine Regelungskompetenz. In Sonderheit gilt dies für den verwaltungsgerichtlichen vorläufigen Rechtsschutz. Für eine durchgreifende Wirkung bei der Vereinheitlichung der Maßstäbe für die Rechtsschutzgewährung hat indessen die Judikatur gesorgt. Dazu stellte der frühere Präsident des Europäischen Gerichtshofs fest, dass die dazu ergangene Rechtsprechung „die intensivste Auswirkung des Gemeinschaftsrechts auf die Verfahrensautonomie der Mitgliedstaaten” bedeutet. ${ }^{*} 129$

\section{a) Richterrechtliche Harmonisierung des vorläufigen Rechtsschutzes}

Die Frage der Europäisierung des vorläufigen Rechtsschutzes ist aufgeworfen, wenn im Rahmen eines Eilantrags bei einem mitgliedstaatlichen Gericht die Gültigkeit der dem nationalen Vollzugsakt zu Grunde liegenden Norm des sekundären Unionsrechts bestritten wird. Nationale Gerichte sind nicht befugt, Handlungen der Unionsorgane für ungültig zu erklären; diese Kompetenz hat allein der Europäische Gerichtshof. ${ }^{* 130}$ Auf der anderen Seite verlangt der effektive Rechtsschutz ${ }^{{ }^{*} 131}$ jedoch die Befugnis der nationalen Gerichte zur Aussetzung der Vollziehung eines auf einer europäischen Verordnung, Richtlinie oder Kommissionsentscheidung beruhenden nationalen Verwaltungsakts, wenn die Gültigkeit der Rechtsgrundlage bestritten ist. ${ }^{*} 132$ Der Gerichtshof hat die Befugnis zur Gewährung vorläufigen Rechtsschutzes anerkannt, behauptet jedoch, dafür müssten in allen Mitgliedstaaten einheitliche Regeln gelten, die denjenigen der Unionsgerichte entsprächen. ${ }^{*} 33$ Der EuGH überträgt also kurzerhand die Voraussetzungen seiner Befugnis zur Aussetzung der Vollziehung einer Verwaltungsmaßnahme (Art. 278 AEUV) auf die nationalen Gerichte.

Danach darf das nationale Gericht die Vollziehung des Verwaltungsakts aussetzen, wenn es

(1) erhebliche Zweifel an der Gültigkeit des sekundären Unionsrechtsaktes hat,

(2) die Frage der Gültigkeit dem Gerichtshof, soweit dieser noch nicht damit befasst ist, vorlegt,

(3) die Eilentscheidung dringlich ist und dem Antragsteller ein schwerer und nicht wiedergutzumachender Schaden droht und

(4) wenn das um vorläufigen Rechtsschutz angerufene nationale Gericht die Interessen der Europäischen Union angemessen berücksichtigt. ${ }^{*}{ }^{134}$

126 Europäische Kommission, Vertragsverletzung Nr. 2007/4267, Stellungnahme vom 26.4.2013 C(2013) 2173 final, S. 11.

127 EuGH, C-115/o9 (Fn. 112), Rdn. 37 und Rdn. 38.

128 EuGH, Urteil v. 7.11.2013, C-72/12, Gemeinde Altrip. - NVwZ 2014, 49 Rdn. 49 anerkennt den subjektiven Rechtswidrigkeitszusammenhang des nationalen Rechts.

129 G. C. Rodríguez Iglesias (Fn. 13), S. 294.

130 EuGH, Urteil v. 21.12.2011, C-366/10, ATA. - NVwZ 2012, 226 Rdn. 47 und 48 (m.w.N.).

131 Vgl. Art. 47 Charta der Grundrechte der Europäischen Union; dazu H. Jarass. Bedeutung der EU-Rechtsschutzgewährleistung für nationale und EU-Gerichte. - NJW 2011, S. 1393ff.

${ }_{132}$ EuGH, Urteil v. 21.2.1991, C-143/88 und C-92/89, Zuckerfabriken Süderdithmarschen. - Slg. 1991, I-532 Rdn. 14ff. (auch in: EuZW 1991, 313; NVwZ 1991, 460); EuGH, Urteil v. 6.12.2005, C-461/o3, Gaston Schul. - Slg. 2005, I-10513 Rdn. 18 (auch in: EuGRZ 2006, 253 Rdn. 18); EuGH, Urteil v. 12.1.2013, C-416/10. - NuR 2013, 113 Rdn. 107.

133 EuGH, Urteil v. 6.12.2005, C-453/o3 u.a., ABNA. - Slg. 2005, I-10423 Rdn. 104 (auch in: EWS 2006, 73 Rdn. 104); EuGH, Urteil v. 13.3.2007, C-432/o5, Unibet. - Slg. 2007, I-2271 Rdn. 79 (auch in: EuZW 2007, 247 Rdn. 79; NJW 2007, 3555 Rdn. 79).

134 EuGH, C-143/88 u.a. (Fn. 132), Rdn. $23 f f$. 
Diese Kriterien für den vorläufigen Rechtsschutz im gerichtlichen Aussetzungsverfahren (§ 80 Abs. 5 VwGO) hat der Gerichtshof auf das Eilverfahren zum Erlass einer einstweiligen Anordnung (§ 123 VwGO) übertragen. ${ }^{*} 135$

\section{b) Kompetenzüberschreitung des EuGH}

Eine Kompetenzgrundlage für seine richterliche Kreation von Entscheidungsmaßstäben für Eilentscheidungen nationaler Gerichte bei Sachverhalten mit EU-Rechtsbezug nennt der Europäische Gerichtshof nicht. Da die Organkompetenz des Gerichtshofs nicht weiter reichen kann als die Verbandskompetenz der Europäischen Union, ist eine Kompetenznorm auch nicht erkennbar. Bemüht wird vielmehr die „Kohärenz des Systems des vorläufigen Rechtsschutzes”. ${ }^{*}{ }^{16}$ Dabei handelt es sich jedoch nicht um eine Kompetenznorm, sondern um ein rechtspolitisches Postulat. ${ }^{*} 137$

Im deutschen rechtswissenschaftlichen Schrifttum wird die skizzierte EuGH-Rechtsprechung weithin als kompetenzwidrig („ultra vires”) eingestuft. ${ }^{* 138}$ Das Bundesverfassungsgericht hat jene Judikatur unter Rechtsschutzaspekten (Art. 19 Abs. 4 GG) akzeptiert; ${ }^{*} 39$ die Kompetenzfrage hat das höchste deutsche Gericht jedoch gar nicht erst aufgeworfen. Europarechtlich ist kritisiert worden, die „Kohärenz”-Argumentation des Gerichtshofs setze die Rechtsprechung zur „Zauberformel” des effet utile fort und solle offenbar von der Beachtung des Gebots der begrenzten Einzelermächtigung (Art. 5 Abs. 1 S. 1, Abs. 2 EUV) entbinden, obgleich in den entschiedenen Fällen zum vorläufigen Rechtsschutz der Äquivalenzgrundsatz und der Effektivitätsgrundsatz zur Sicherung des einheitlichen Vollzugs des EU-Rechts ausgereicht hätten. ${ }^{*} 140$ Dieser zutreffenden Einschätzung ist nichts hinzuzufügen.

\section{Fazit und Ausblick}

Die Europäisierung des Verwaltungsrechts ist zu einer unüberwindbaren Determinante der modernen Staatsverwaltung geworden. Keine innerstaatliche Stelle kann sich den Einwirkungen des Unionsrechts entziehen. Über ihre Einhaltung durch die Mitgliedstaaten wacht der Europäische Gerichtshof. Er hat Instrumente entwickelt, die auf die Durchsetzung und reale Geltung des Unionsrechts zielen. Das ist hier an drei übergreifenden Rechtsgebieten (Allgemeines Verwaltungsrecht, Verwaltungsverfahrensrecht, Verwaltungsprozessrecht) skizziert worden. Im Besonderen Verwaltungsrecht ist die Europäisierung zum Teil noch weiter fortgeschritten. ${ }^{*} 141$ Die Mitgliedstaaten sind aufgerufen, diesen Prozess der Durchdringung innerstaatlichen Rechts durch das Unionsrecht ernst zu nehmen und, wo dies notwendig ist, kritisch zu begleiten. Dabei kommt der entsprechenden Ausbildung junger Juristen und der Weiterbildung im Beruf befindlicher Juristen ein hoher Stellenwert zu. Denn nur wer über die Europäisierung des Verwaltungsrechts informiert ist, kann als Jurist die Zeichen der Zeit erkennen.

\footnotetext{
135 EuGH, Urteil v. 9.11.1995, C-465/93, Atlanta. - Slg. 1995, I-3781 Rdn. 32 ff. (auch in: EuZW 1995, 837 Rdn. 32 ff.; NJW 1996, 1333 Rdn. 32 ff.); EuGH, Urteil v. 5.12.1996, C-69/95, T. Port. - Slg. 1996, I-6065 Rdn. 49 ff. (auch in: NJW 1997, 1225 Rdn. $49 f f$.$) .$

136 EuGH, C-143/88 u.a. (Fn. 132), Rdn. 18, 27; EuGH, C-465/93 (Fn. 135), Rdn. 22, 39; EuGH, C-453/o3 u.a. (Fn. 133 ), Rdn. 103.

137 F. Schoch. - W. Hoffmann-Riem, E. Schmidt-Aßmann, A. Voßkuhle (Hrsg.) (Fn. 24), § 50 Rdn. 26ff. (mit umfangreichen Nachweisen).

${ }_{138}$ Nachweise dazu bei F. Schoch. - F. Schoch, J.-P. Schneider, W. Bier (Hrsg.) (Fn. 12), § 8o Rdn. 393 und 394.

139 BVerfG, Beschluss v. 27.7.2004, 1 BvR 1270/04. - NVwZ 2004, 1346 (1347).

140 R. Streinz. Primär- und Sekundärrechtsschutz im Öffentlichen Recht. - VVDStRL 2002, S. $342 f$.

${ }_{141}$ Beispiele: Umweltrecht, Vergaberecht, Lebensmittelrecht, Umweltinformationsrecht, Wirtschaftsverwaltungsrecht.
} 